\title{
RESISTANCE À LA FATIGUE D'ACIERS DE CONSTRUCTION TRAITÉS PAR LASER
}

\author{
M. THÉOBALT, P. MERRIEN*, F. LEROY**, J.L. LEBRUN*** et T. PUIG**** \\ IRSID Unieux, B.P. 50, F-42702 Firminy, France \\ ${ }^{*}$ CETIM, 52 av. Felix Louat, F-60300 Senlis, France \\ **ASCOMETAL, Laboratoire d'Usinabilité et de Mise en Forme, F-42702 Firminy, France \\ ***ENSAM, 151 bd de l'Hôpital, F-75013 Paris, France \\ ****ETCA, rue de la Côte d'Or, F-94114 Arcueil cedex, France
}

\begin{abstract}
In order to improve the mechanical properties, and thus the in-service behaviour, of mechanical components subjected to fatigue and wear type working conditions the automobile industry uses surface treatment techniques.

Using the $\mathrm{CO}_{2}$ continuous wave LASER as a surface treatment method allows mechanical components, which are subsequently subjected to high loading levels, to be locally and preferentially hardened. The advantage of this technique lies in its flexibility. Such a tool could easily be integrated into a production line, not only allowing on-line treatment but also considerably reducing the component downtime and stock level. The results of the present study have shown that the fatigue endurance limit obtained for specimens treated superficially by laser was comparable to that determined for specimens subjected to a carbonitriding treatment. In order to attain such a result several parameters were optimised including i) the partition of the laser beam's energy, power and the test piece treatment time ; ii) pre-treatment preparation of the test piece surface ; iii) initial metallurgical state of the material to be treated.

In order to examine and quantify the fatigue behaviour of the laser treated material, both metallurgical (structure, hardness) and the residual stress fields were analysed. For the quenched and tempered $42 \mathrm{CD} 4$ steel examined, a laser surface treatment resulted in fatigue properties which were similar to those obtained for specimens subjected to a carbonitriding treatment under industrial conditions.
\end{abstract}




\section{1 - Introduction}

Les pièces en aciers de construction utilisées dans l'industrie automobile sont soumises à des chargements très élevés souvent localisés (pied de dent de pignons, congés de raccordement dans les vilebrequins). L'emploi des traitements de surface industriels actuels (- thermochimiques : nitruration, cémentation, carbonitruration - thermiques rapides : trempe H.F.) permet d'augmenter de façon substantielle les caractéristiques des matériaux utilisés, notamment en ce qui concerne leur résistance à la fatigue, dans les zones soumises à de fortes concentrations de contraintes.

L'objectif de ce travail est d'étudier la faisabilité d'un traitement de durcissement superficiel par "trempe Laser", dans des conditions de laboratoire, et de tester les performances en fatigue du matériau traité après optimisation du procédé et de la métallurgie initiale, afin de confronter ce procédé avec un traitement plus classique utilisé par les constructeurs : la carbonitruration.

\section{2 - le traitement de trempe Laser}

Les sources Laser sont actuellement utilisées dans l'industrie pour un large éventail d'applications, selon les gammes de puissances et de temps d'interaction Laser / matière.

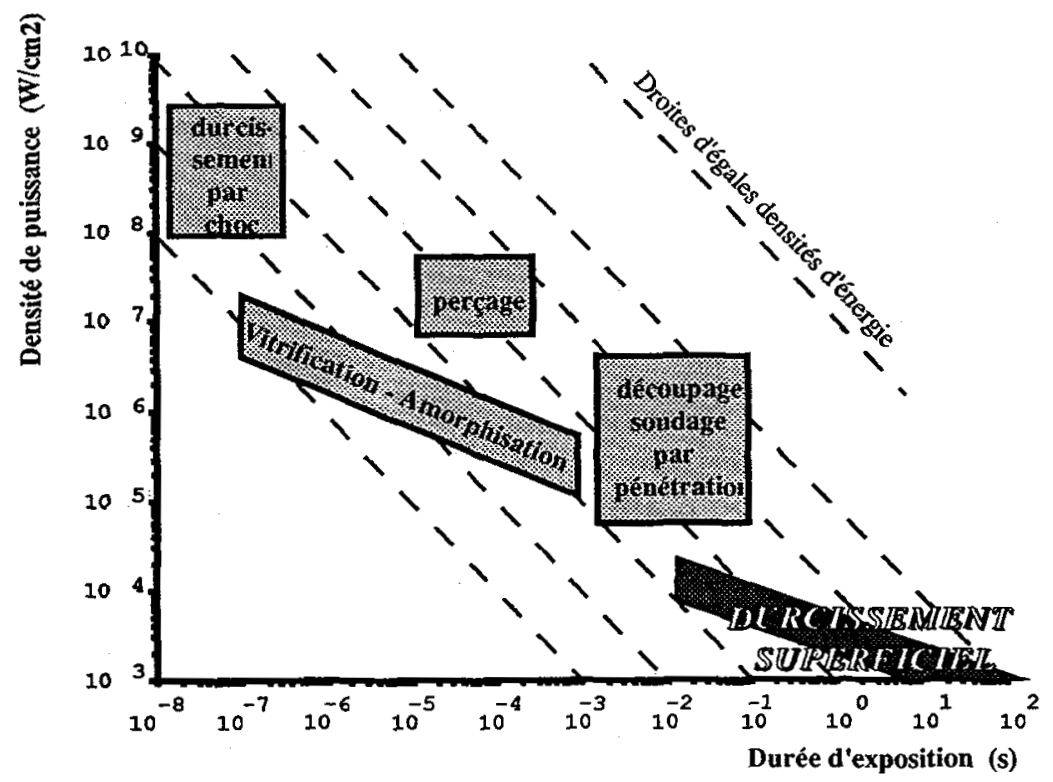

Figure 1 : Applications des Lasers selon les gammes de puissances et de temps d'interaction [1].

Un traitement de durcissement superficiel nécessite une densité de puissance de l'ordre de quelques $\mathrm{kW}$, pendant une durée de l'ordre de la seconde. 
Le durcissement superficiel par faisceau LASER consiste à porter localement le matériau à une température supérieure à la température d'austénitisation avec une vitesse de chauffage élevée [2] $\left(2.10^{3}{ }^{\circ} \mathrm{C} / \mathrm{s}\right.$ à $\left.2.10^{\circ} \mathrm{C} / \mathrm{s}\right)$ sans atteindre la température de fusion. Le temps d'exposition au faisceau est relativement court, typiquement de l'ordre de la seconde. Compte tenu des températures élevées atteintes localement $\left(>1000^{\circ} \mathrm{C}\right)$, et de la faible quantité de chaleur transmise à la pièce, le refroidissement de la zone traitée se fait par conduction dans la masse de la pièce (autotrempe), et ce à une vitesse supérieure à la vitesse critique de trempe du matériau pour permettre la formation d'une structure de trempe superficielle (martensite fine). Il y a donc trempe dans la zone que l'on souhaite traiter aux deux conditions suivantes :

i) le matériau est porté à une température supérieure à la température d'austénitisation.

ii) la vitesse de refroidissement est supérieure à la vitesse critique de trempe propre du matériau.

Les traitements ont été effectués avec l'installation de l'ETCA à l'aide d'un LASER continu $\mathrm{CO}_{2}$ d'une puissance de $5 \mathrm{~kW}$. Le faisceau a fait l'objet d'une optimisation de sa répartition énergétique, à l'aide d'un kaleidoscope constitué de miroirs de cuivre, afin d'obtenir au niveau de la cible une densité d'énergie constante et homogène sur $10 \times 10 \mathrm{~mm}^{2}$.

Les lasers $\mathrm{CO}_{2}$ émettent un rayonnement de longueur d'onde $\lambda=10,6 \mu \mathrm{m}$. Un tel rayonnement est quasiment intégralement réfléchi par l'acier ; il est par conséquent nécessaire d'effectuer un dépôt préalable sur la pièce à traiter de façon à augmenter son coefficient d'absorbtion pour une telle longueur d'onde.

Les dépôts tels que le phosphate de manganèse, le graphite ou la peinture noire possèdent un très bon coefficient d'absorbtion vis à vis de la longueur d'onde du laser $\mathrm{CO}_{2}$ [3]. Le procédé ici utilisé est une phosphatation sèche au manganèse qui conduit à un coefficient d'émissivité de 0,8 avec une bonne reproductibilité.

\section{Matériaux}

Nous avons étudié les effets de l'autotrempe par faisceau Laser sur la tenue en fatigue d'un acier $42 \mathrm{CD} 4(0,425 \mathrm{C} ; 0,740 \mathrm{Mn} ; 0,260 \mathrm{Si} ; 0,013 \mathrm{P} ; 0,020 \mathrm{~S} ; 0,267 \mathrm{Ni} ; 0,965 \mathrm{Cr} ; 0,142 \mathrm{Mo})$ à partir d'observations sur la dureté, la microstructure et la répartition des contraintes résiduelles dans le matériau.

La nuance $42 \mathrm{CD} 4$ est étudiée à l'état trempé revenu (homogénéisation à $850^{\circ} \mathrm{C}$ pendant $1 / 2 \mathrm{~h}$. suivi d'un refroidissement huile + revenu à $550^{\circ} \mathrm{C}-\mathrm{TTA}-, 600^{\circ} \mathrm{C}-\mathrm{TTB}-, 650^{\circ} \mathrm{C}-\mathrm{TTC}-$, pendant 2 h.) avec une structure métallurgique martensitique revenue. 
Les conditions du traitement Laser ont été optimisées de sorte que la profondeur traitée soit de l'ordre de $0,7 \mathrm{~mm}$ (puissance du faisceau à l'émission : $\mathbf{P}=2,4 \mathrm{~kW}$; temps d'interaction Laser / matière : $t=1,3 \mathrm{~s}-1,8 \mathrm{~s}$ ). Le traitement de la pièce se fait de façon statique sous balayage d'argon.

4 - Caractéristiques de la zone traitée.

\section{4- 1 Micrographie - Dureté}

La zone traitée présente en surface une martensite très fine et homogène. La zone de transition est constituée d'une structure bainito-martensitique.

Les mesures de dureté montrent que la profondeur durcie est d'autant plus grande que le temps d'interaction du faisceau avec le matériau est long. Par ailleurs, la profondeur traitée est plus importante, toutes conditions de traitement égales, sur une nuance trempée revenue à $550^{\circ} \mathrm{C}$ plutôt qu'à $650^{\circ} \mathrm{C}$.

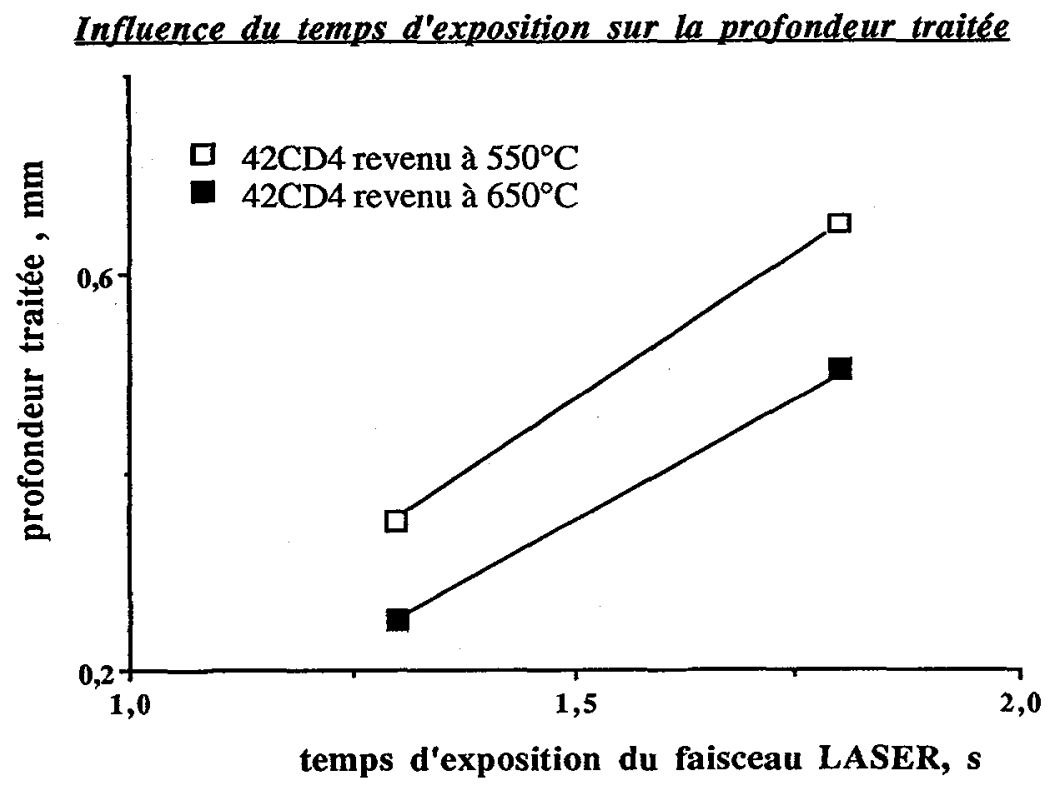

Figure 2 : Influence du temps d'exposition sur la profondeur traitée. 


\section{4 - 2 Contraintes résiduelles}

La détermination des contraintes résiduelles a été effectuée à l'ENSAM-PARIS par diffraction $X$ sur un appareil SET-X. La mesure de la position du pic, qui traduit l'intensité des contraintes macroscopiques dans le matériau, est accompagnée d'une mesure de la largeur intégrale du pic (aire divisée par l'intensité) exprimée en $\mathrm{nm}^{-1}$. Cette grandeur permet de caractériser l'état de microdéformation du matériau (taux de dislocations, carbone interstitiel).

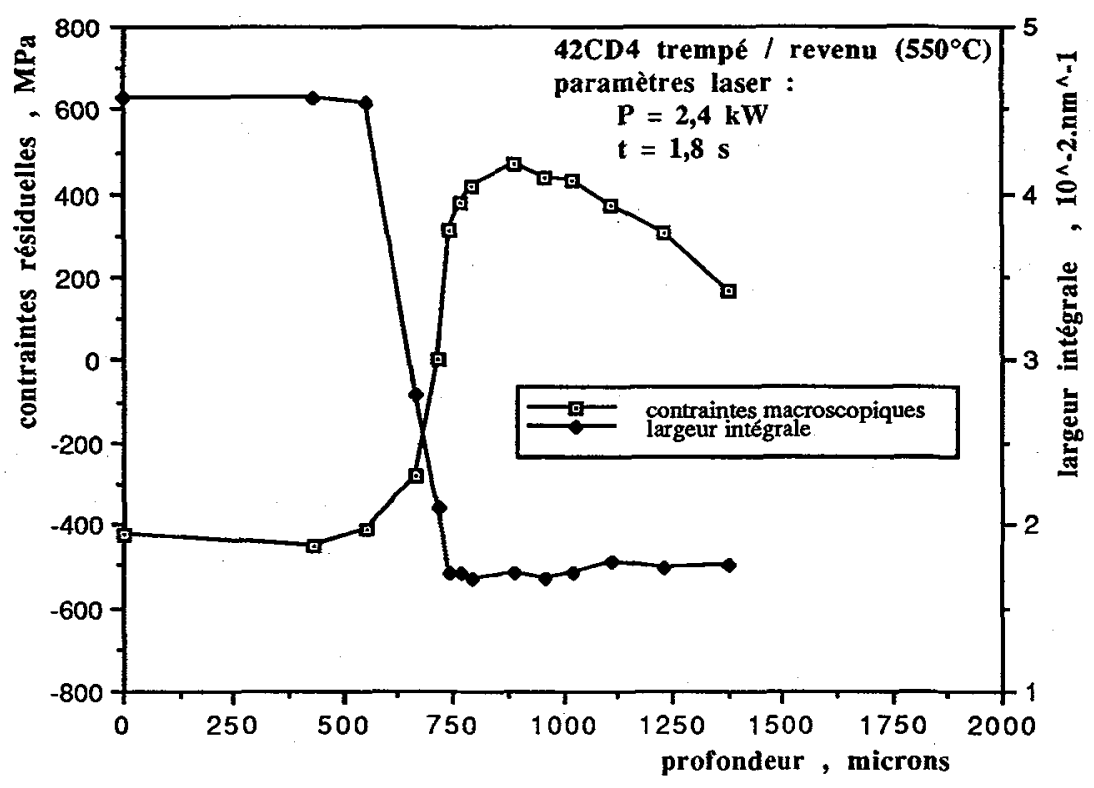

Figure 3 : Profil de contraintes résiduelles et de largeur de corde

dans la zone traitée sur la nuance $42 \mathrm{CD} 4$ trempée revenue à $550^{\circ} \mathrm{C}$ traitée dans les conditions suivantes : $P=2,4 \mathrm{~kW} ; t=1,8 \mathrm{~s}$.

Sur toute la profondeur de la zone durcie, les éprouvettes présentent des contraintes de compression élevées. Les mesures de largeur intégrale élevée traduisent un écrouissage important de cette zone, correspondant à une martensite très dure. Au delà, la zone de transition avec le métal de base est caractérisée par la présence d'un pic de traction et par une diminution sensible de la largeur de corde. Sachant que les fissures de fatigue sur 42CD4 traité par trempe LASER s'amorcent généralement en sous couche, ce sont les caractéristiques locales du matériau dans cette zone qui gouverneront sa tenue à la fatigue. 
Les paramètres tels que le temps d'exposition de la pièce au faisceau Laser, ou la métallurgie du matériau de base (due au revenu d'élaboration) sont à l'origine des caractéristiques - profondeur, intensité - du pic de traction.

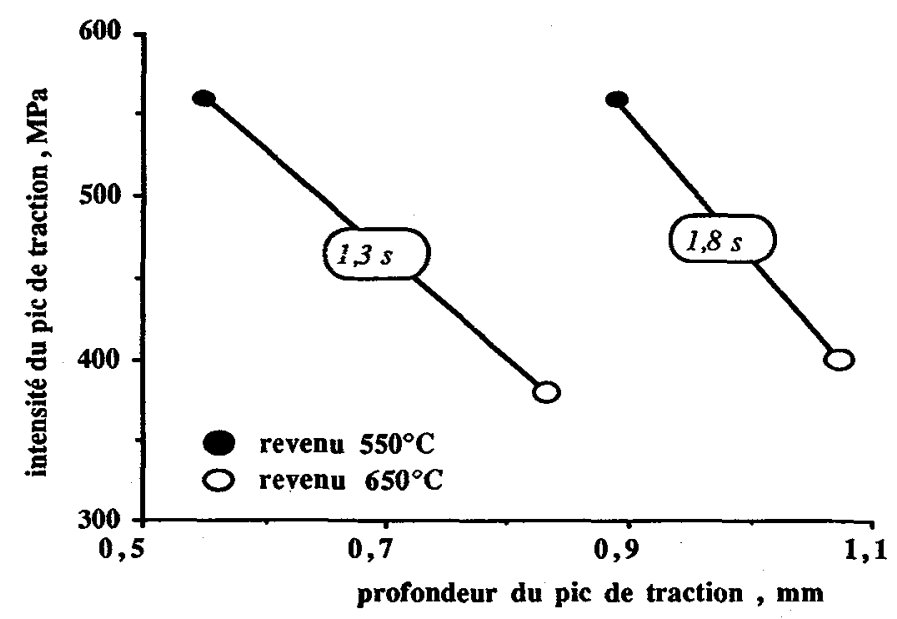

Figure 4 : Influence du temps d'exposition et du revenu d'élaboration sur le pic de traction en sous couche.

\section{5- Résultats de fatigue}

Les essais de fatigue sont réalisés en flexion 3-points sur barreaux entaillés. L'entaille, qui constitue la partie utile de la pièce, reproduit un coefficient de concentration de contrainte $\mathrm{Kt}=1,65$. Au cours de la trempe Laser, seule l'entaille est traitée.

Selon les paramètres de traitement, la limite de fatigue du matériau est améliorée de $25 \%$ à $45 \%$ après trempe LASER. Les résultats de fatigue du 42CD4 traité par LASER (Kt $\Delta \sigma$ compris entre $1085 \mathrm{MPa}$ et $1263 \mathrm{MPa}$ selon les paramètres de traitement) sont comparables à ceux obtenus sur un $27 \mathrm{CD} 4$ après carbonitruration (Kt $\Delta \sigma$ compris entre $1050 \mathrm{MPa}$ et $1250 \mathrm{MPa}$ selon les traitements de carbonitruration). Par ailleurs, la dispersion des résultats de fatigue obtenus sur cette nuance 42CD4 traitée par trempe LASER reste beaucoup plus faible (écart type inférieur à 30MPa) à ce qui est couramment obtenu après carbonitruration. 
Tableau 1 : Résultats de fatigue sur 42 CD4 traité par Laser.

\begin{tabular}{|c|c|c|c|c|}
\hline nuance & $\begin{array}{c}\text { température du revenu } \\
\text { d'élaboration }\end{array}$ & \multicolumn{2}{|c|}{$\begin{array}{l}\text { conditions } \\
\text { de traitement }\end{array}$} & $\begin{array}{l}\text { limite de } \\
\text { fatigue (MPa)* }\end{array}$ \\
\hline $42 \mathrm{CD} 4$ & $\begin{array}{l}\mathrm{TTA}=550^{\circ} \mathrm{C} \\
\text { TTB }=600^{\circ} \mathrm{C} \\
\text { TTC }=650^{\circ} \mathrm{C} \\
\text { métal de base }\end{array}$ & $\begin{array}{c}\text { trempe } \\
\text { Laser }\end{array}$ & $\begin{array}{l}P=2.4 \mathrm{~kW}, t=1.8 \mathrm{~s} \\
P=2.4 \mathrm{~kW}, \mathrm{t}=1.3 \mathrm{~s} \\
P=2.4 \mathrm{~kW}, \mathrm{t}=1.8 \mathrm{~s} \\
P=2.4 \mathrm{~kW}, \mathrm{t}=1.8 \mathrm{~s} \\
P=2.4 \mathrm{~kW}, \mathrm{t}=1.3 \mathrm{~s}\end{array}$ & $\begin{array}{c}1125 \pm 30 \\
1118 \pm 11 \\
1263 \pm 13 \\
1268 \pm 11 \\
1085 \pm 45 \\
870 \pm 7\end{array}$ \\
\hline 27CD4 & & \multicolumn{2}{|c|}{ carbonitruration } & $1050 / 1250$ \\
\hline
\end{tabular}

* les résultats de fatigue sont exprimé en termes d'amplitude de contrainte à fond d'entaille, c'est à dire $\mathrm{Kt}$ x $\Delta \sigma_{\text {nominale }}(\mathrm{Kt}$ : coefficient de concentration de contrainte ).

Afin d'interpréter les résultats de fatigue, il convient de tenir compte simultanément des paramètres métallurgiques (précipités, interstitiels, taux d'écrouissage) facteurs de durcissement traduits par la largeur de corde -, et des contraintes résiduelles macroscopiques [4][5].

Les modèles prévisionnels actuels de tenue en fatigue (critères multiaxiaux), pour des pièces comportant de telles couches traitées, intégrent le champ des contraintes résiduelles induites dans le matériau ainsi que le durcissement superficiel. Ces modélisations nécessitent néanmoins un ajustement de certains coefficients en fonction des résultats expérimentaux obtenus par ailleurs sur des matériaux identiques. En effet, le paramètre "durcissement" n'intervient pas systématiquement de la même manière selon les matériaux étudiés [4].

A partir des résultats présentés ci-dessus, il est toutefois possible de faire les remarques suivantes :

- La limite de fatigue augmente avec la profondeur traitée (c'est à dire avec le temps d'interaction Laser / matière) sauf dans le cas d'un revenu d'élaboration à $550^{\circ} \mathrm{C}$ auquel cas la limite de fatigue reste constante.

- Les meilleurs résulats de fatigue sont obtenus sur le matériau ayant subi un revenu d'élaboration de $600^{\circ} \mathrm{C}$ ou $650^{\circ} \mathrm{C}$, traités avec un temps d'interaction de $1,8 \mathrm{~s}$, conduisant à une profondeur traitée de l'ordre de $0,7 \mathrm{~mm}$. 
- Lorsque l'amorçage intervient en surface, l'intensité des contraintes résiduelles superficielles de compression jouent un rôle déterminant dans l'augmentation de la limite d'endurance du matériau traité.

- Lorsque l'amorçage intervient en sous-couche, comme c'est le cas ici, les contraintes résiduelles macroscopiques dans cette zone (pic de traction), et le taux de microdéformation local, influent simultanément sur la résistance à la fatigue. Globalement, il est possible de dire que la limite de fatigue du matériau traité localement augmente lorsque :

* la profondeur à laquelle se trouve le pic de traction augmente.

* lintensité du pic de traction diminue.

* l'adaptabilité du matériau à la déformation dans la zone de transition augmente.

* la résistance du matériau dans la zone de transition augmente.

Pour pouvoir apprécier ces deux derniers paramètres indépendemment, il conviendrait de pouvoir déconvoluer ce qui, dans la largeur de corde mesurée dans la zone de transition, tient de l'écrouissage du matériau, et ce qui tient du surrevenu métallurgique (précipitation, coalescence des précipités, taux de $\mathrm{C}$ moyen dans la matrice).

\section{6 - Conclusions}

Cette étude montre qu'au terme d'une optimisation des conditions expérimentales (répartition de la densité énergétique du faisceau, optimisation de l'absorbtivité des surfaces à traiter à l'aide d'un dépôt de phosphate de $\mathrm{Mn}$ ) le traitement par faisceau Laser permet d'obtenir localement sur du $42 \mathrm{CD} 4$ des structures de trempe (martensite fine) caractérisées par une dureté importante et des contraintes résiduelles superficielles de compression.

Ces modifications s'accompagnent d'une augmentation substantielle de la limite de fatigue du matériau en flexion 3-points. Les résultats obtenus sont similaires à ceux obtenus au terme d'un traitement de carbonitruration dans des conditions industrielles.

Pour ce qui est du choix d'une métallurgie de base (température de revenu d'élaboration), les résultats sont optimisés en trouvant, en cas de rupture sous la couche traitée, un compromis entre une résistance élevée du matériau de base, et une bonne capacité à l'accommodation des déformations au voisinage des inclusions dans la zone affectée par le surrevenu métallurgique.

\section{REFERENCES}

[1] GUESLOT-MARTIN, "Application des Lasers de Forte Puissance", CETIM Information n 88, décembre 1984.

[2] MARUO, "Effect of Heating Conduction in Laser Hardenning of Carbon Steel", Proceedings of the IRST Laser Processing Conference (ANAHEIM, USA), november 1986. 
[3] MEGAW, "High Power Lasers and Laser Metalworking SPIE, vol 164, Fourth European Electro-optics conference (SIRA, oct. 1978, UTRECHT, Netherlands).

[4] A. DEPERROIS, L. CASTEX, K. DANG VAN, P. MERRIEN, A. BIGNONNET, "High Cycle Fatigue Life of Surface Hardened Steels Under Multi-axial Loading", Fatigue 90, HONOLULU, vol 4, pp. 2149-2154.

[5] J. BARRALIS, L. CASTEX, "Improvement of Fatigue Resistance of Steel Parts after Various Surface Treatments", Surface Engineering, Editors S.S. MEGUID, Elsevier Applied Science 1990, pp. 625-639. 